\title{
LOS ECONOMISTAS BAJO LA ESVÁSTICA
}

\author{
FERNANDO LEAL CARRETERO
}

Universidad de Guadalajara. México

\author{
PALABRAS CLAVE ADICIONALES \\ ADDITIONAL KEYWORDS \\ Nazismo, Historia económica, Alemania. \\ Nazism, Economic History, Germany.
}

\begin{abstract}
La RIS tiene la satisfacción de incluir en su sección de Personalia un ensayo del profesor mexicano Fernando Leal Carretero en el que analiza el trabajo de los economistas alemanes durante un periodo histórico de gran trascendencia, como fue el protagonizado por el partido nacionalsocialista en Alemania en los años 30 y 40 del pasado siglo XX.
\end{abstract}

El profesor Fernando Leal Carretero es actualmente catedrático de epistemologia y metodologia de las ciencias sociales en la Universidad de Guadalajara (México). Realizó estudios de filosofia, lingüistica general y filologia clásica en las universidades alemanas de Heidelberg, Bonn y Colonia, doctorándose en 1983. Ha sido investigador visitante en las universidades de Colonia y Londres y ha publicado un centenar de artículos en revistas internacionales o capitulos en libros. Es coeditor de los libros siguientes: Person-centered Ergonomics: the Brantonian View of Human Factors (Londres, 1993), Ethics and the New Technology (Londres y Berlin, 1995) $e$ Introducción al estudio del español desde una perspectiva multidisciplinaria (Guadalajara, 2003). Sus áreas de trabajo son filosofia del lenguaje, ética y teoria general de los valores, filosofia y metodologia de las ciencias sociales y cognitivas, economia politica, teoria lingüistica general, y lingüistica aplicada al estudio de las trastornos en el desarrollo del lenguaje.

E-mail: f.leal@Prodigy.net.mx

Revista Internacional de Sociología (RIS)

Tercera Época, № 35, Mayo-Agosto, 2003, pp. 201-220. 


\begin{abstract}
No habrá licencias, no habrá espacios libres en que el individuo se pertenezca a si mismo. Esto es socialismo - no tonterias como la propiedad privada de los medios de producción-. ¿Qué importancia tiene eso si yo puedo meter a la gente firmemente dentro de una disciplina de la que no pueda escapar? Déjelos que sean dueños de cuanta tierra y cuantas fábricas quieran. El factor decisivo es que el Estado, a través del partido, reina supremo sobre ellos, sean ellos dueños o trabajadores. Verá usted, todo eso no es esencial. Nuestro socialismo va más hondo... ¿Para qué molestarse en socializar los bancos y las fábricas? Nosotros socializamos la persona entera. (Hitler)

Lo único que nos importa y para lo que les pagamos a esa gente [los historiadores], son imágenes históricas que le refuercen a nuestro pueblo el necesario orgullo nacional. El único interés que tenemos en esta dudosa empresa es que la imagen futura que nos hacemos de nuestro pueblo se proyecte al pasado inmemorial. Todo Tácito y su Germania es un escrito propagandístico. Toda nuestra ciencia de la germanidad ha vivido durante siglos de una falsificación. Tenemos el derecho de repetir estas cosas una y otra vez. La prehistoria es la doctrina de la inmensa importancia de los alemanes en el pasado. (Göring)'
\end{abstract}

Mucho se ha hablado del carácter internacionalista del socialismo en general y del movimiento obrero en particular. En mi juventud estaba convencido de todos esos cuentos de hadas. Tanto más triste y doloroso fue mi desengaño cuando me tocó oír a un famoso líder sindical alemán hablar (allá por 1982) de cómo los obreros del Tercer Mundo les "robaban" los puestos de trabajo a los alemanes: una de las consecuencias, según él, de la instalación de plantas industriales en Asia, África y América Latina. Entre el público había estudiantes de varias nacionalidades, y la reacción a la perorata desvergonzadamente nacionalista de aquel líder era distinta según el lugar de origen de quienes la escuchábamos. No sé qué me deprimió más, si oír el discurso o ver cómo mis compañeros alemanes - tan de izquierdas como el que más - se quedaban tan anchos, sin percibir ningún motivo de escándalo. No fue sino mucho tiempo después que comencé a entender las razones de todo ello.

El nombre griego "economía" (oikonomía) designa la administración de la hacienda (oikós), es decir el conjunto de reglas y principios (nómoi) que deben seguirse para que la propia hacienda produzca el mayor número de bienes de la manera más eficiente. La nueva ciencia que en el siglo XVIII emerge de los escritos de Richard Cantillon, David Hume, François Quesnay, Adam Smith, Etienne Condillac y Jacques Turgot, de manera natural adopta ese viejo nombre griego, pero lo califica con el adjetivo "política", igualmente griego, y que designa justamente una unidad de civilización (pólis) cuya productividad interesa optimizar. Pero,

\footnotetext{
${ }^{1}$ Palabras atribuidas a Hitler y Göring por Hermann Rauschning en su conocido libro Gespräche mit Hitler (1940). La primera cita se tradujo de la versión inglesa (The Voice of Destruction, Nueva York, Putnam, 1940, pp. 191-193); la segunda se reproduce en el libro que nos ocupará principalmente en este trabajo (Janssen, 2000: 151-152).
} 
¿cuál es la unidad de civilización relevante? Dada la preeminencia de ese notable invento de la era moderna, el Estado nación, pareciera que tal y no otra debe ser la unidad a estudiar: la economía política tendría que ser economía nacional.

Sin embargo, las cosas no son así de sencillas. He aquí que la economía política nace con el liberalismo, uno de cuyos pilares es la idea del comercio libre, y ello justamente porque - tal es el resultado cada vez más firme de la nueva ciencia-el mercado libre parece ser el único mecanismo capaz de garantizar la mayor riqueza, pero no para una sola nación, sino para todas. El Estado nacional que se cierre al comercio con las demás naciones -nos dicen y repiten los economistas políticos- será menos productivo y más pobre. La verdadera economía política sería, por tanto, no economía nacional, sino economía cosmopolita, economía del mundo entero.

Estas dos grandes visiones de la nueva ciencia - ciencia de la riqueza de un Estado nacional vs. ciencia de la riqueza de todo el mundo- se enfrentan en una disputa que lleva más de dos siglos y no da ninguna señal de abatirse. La pequeña anécdota con la que inicié este trabajo es sólo un botón de muestra: mi internacionalismo ingenuo me hacía pensar de manera cosmopolita, pero a aquel líder sindical le preocupaba solamente la riqueza de su país.

Pues bien, el reciente libro de Hauke Janssen trata de uno de los capítulos más curiosos y a la vez menos conocidos de la disputa entre economía nacional y economía cosmopolita: el comportamiento de los economistas alemanes entre 1933 y $1940^{2}$. Dado el carácter furibundamente nacionalista del tercer Reich es obvio que la economía política que le corresponde sea una ciencia nacional, no cosmopolita. Pero el carácter histórico que la nueva ciencia adoptó en suelo alemán en el periodo que va desde la fundación de la nueva ciencia hasta la llegada al poder de los nazis - digamos desde Friedrich List hasta Werner Sombart- fue de manera preponderante (aunque de ninguna manera exclusiva, lo cual tiene su importancia, como se verá más adelante) el de una economía nacional; de hecho, el nombre más popular para la nueva ciencia en Alemania en ese período fue justamente Nationalökonomie. Luego, se suscita la pregunta de si tal carácter nacionalista estaba, por decirlo así, predestinado a que los economistas alemanes pactasen con, por no decir que se vendiesen a, el régimen de Hitler.

Es en efecto un lugar común decir que esto, tristemente, fue lo que ocurrió. A ese lugar común hace referencia el autor al comenzar el primer capítulo:

En la literatura sobre el nacionalsocialismo se afirma una y otra vez que el desarrollo que llevó al nacionalsocialismo habría sido un desarrollo típicamente alemán, y que, por tanto, en la toma de poder de Hitler habría que "ver el punto de culminación

\footnotetext{
${ }^{2}$ Hauke Janssen, Nationalökonomie und Nationalsozialismus: die deutsche Volkswirtschaftslehre in den dreissiger Jahren, $2^{\mathrm{a}}$ edición, Marburgo, Metropolis-Verlag, 2000, 716 pp.
} 
RIS

REVISTA INTERNACIONAL DE SOCIOLOCIA

N 35, Mayo-Agosto, 2003

FERNANDO LEAL CARRETERO

lógico de la historia alemana" (Janssen, p. 13; la cita remite a la traducción alemana de George Mosse, The Crisis of German Ideology: Intellectual Origins of the Third Reich, Nueva York, 1964).

Sin embargo, como el lector ya se imaginará, las cosas nunca son tan sencillas como parecen. El objetivo del libro de Janssen es mostrarnos cuán complicadas son de verdad cuando se mete uno a hurgar en los detalles de "lo que realmente pasó".

La voluminosa obra de Janssen contiene cinco capítulos de tamaño muy desigual (poco más de 500 páginas), un apéndice (casi 100 páginas) y una abundante lista de referencias bibliográficas (aprox. 60 páginas). Fue originalmente publicada en 1999 y se agotó rápidamente, por lo que un año después se publicó la segunda edición revisada, que es a la que me refiero en este trabajo. El apéndice contiene una especie de Who's Who de la doctrina económica en el mundo de habla alemana entre 1870 y 1940 , el cual es sumamente útil para orientarse prosopográficamente en un libro como éste. El primer capítulo (pp. 13-26) es una breve discusión del estado de la cuestión, donde el autor repasa las principales posturas; el quinto y último reúne las conclusiones de manera apretadísima (pp. 537-539). Entre estos dos breves textos se presentan (bajo la forma de los capítulos 2, 3 y 4) los tres estudios monográficos que constituyen el corazón de la obra, y cuyo propósito y conclusiones procedo a describir por separado ${ }^{3}$.

\section{PRIMER ESTUDIO}

\section{La doctrina económica alemana en los años 20 (Janssen, cap. 2, pp. 27-152)}

El propósito fundamental de este primer estudio es describir el estado de la doctrina económica alemana en los años 20 , es decir, justo en el momento del ascenso del movimiento nacionalsocialista; pero describirlo no tal como se lo imagina el lugar común que se mencionó antes, ni tal como lo presentó la propaganda nazi, sino tal como realmente fue. En efecto, una frase resume gráficamente el principal resultado del primer estudio: "aceptar sin examen la manera en que los nazis presentan la historia del pensamiento económico alemán es ser víctimas, una vez más, de su propaganda" (p. 137).

Dicho brevemente, la escuela histórica alemana y su vieja postura nacionalista se encontraban en los años 20 en pleno declive y a la defensiva frente a los

\footnotetext{
${ }^{3}$ La gran riqueza del libro de Janssen reside, en mi opinión, en el gran número de artículos y libros de teoría y política económica que describe, reseña y sitúa en su contexto histórico. Es imposible hacer justicia a esa riqueza en el espacio de este trabajo.
} 
representantes de la teoría clásica. Es cierto que si uno examina la historia del pensamiento económico alemán a lo largo del siglo XIX encuentra más autores historicistas y nacionalistas que teóricos y cosmopolitas (aunque el conjunto formado por estos últimos no es vacío y cuenta entre ellos con notables pensadores). Sin embargo, esta preponderancia estaba exhausta y no había producido los resultados prometidos. ¿Cuáles eran estos resultados? Esencialmente uno: la crítica a la economía clásica (y neoclásica) que los historicistas e institucionalistas (tanto en Alemania como en otros países, aunque Janssen no habla sino de Alemania) se reducía a objetar la idea de una teoría económica general, válida para todos los tiempos y lugares. Para afianzar semejante crítica era necesario, por tanto, producir una teoría alternativa. Los historicistas decían tener el método correcto (justamente histórico) para encontrar esa nueva teoría. Sin embargo, tras decenios y decenios de acumular datos al mejor estilo inductivista baconiano, esa nueva teoría no había emergido.

Dicho sea de paso, la historia del desarrollo de una teoría, si no literalmente alternativa y opuesta a la teoría neoclásica, al menos sí diferente en algunos puntos de importancia, y que hace justicia a parte de la visión que movia a los viejos historicistas e institucionalistas, no se agota en el episodio sórdido que es el objeto de la narrativa de Janssen. Disciplinas como el análisis económico del derecho, la teoría de los costos de transacción, la economía de la información y el llamado neoinstitucionalismo, entre otras, son ejemplos vivos de que las preguntas de aquellos autores tenían sentido. No se trata, pues, de condenar simplemente a la vieja escuela histórica alemana por haber pensado que la economía clásica no contenía todas las respuestas.

Comoquiera que ello sea, en la Alemania universitaria de los años 20 la desesperación cundía entre los nacionalistas debido a su propia impotencia teórica. Y esta situación se veía, además, exacerbada por los embates de la teoría cosmopolita, reformada y mejorada merced a los nuevos conceptos marginalistas (Jevons, Walras, Menger, Pantaleoni, Pareto, Marshall, Edgeworth, Fisher, Auspitz y Lieben, Wicksell, Böhm-Bawerk, Clark, Wicksteed, etc.). Pero considere el lector la siguiente pregunta: si el antiguo predominio del historicismo nacionalista estaba desapareciendo y la escena doctrinal en Alemania era bastante plural (Janssen nos muestra que ni siquiera la oposición entre los clásicos y los historicistas agota la diversidad real), ¿cómo se ha podido afirmar que el enfoque económico del nacionalsocialismo constituye la culminación lógica del desarrollo del pensamiento alemán en economía?

Para entender esto necesitamos hacer un análisis político de lo que a primera vista parece ser mera historia de las ideas. La idea de que el historicismo nacionalista tradicional culmina en el nacionalsocialismo es el producto del "confuso contubernio" entre dos tipos de actores, cada uno con su agenda política. Por un lado tenemos a los académicos (profesores, asistentes, estudiantes) asociados a la visión nacionalista en retirada y a la defensiva, viendo en peligro sus puestos, 
RIS

salarios y canonjías; por el otro, tenemos a los nazis, una banda de criminales políticos sin el menor escrúpulo. Fue una combinación explosiva. Los primeros querían conservar sus puestos y aumentar su influencia frente a la amenaza de los teóricos cosmopolitas, los segundos buscaban una legitimación histórica de sus políticas ultranacionalistas. Se dieron mutuamente lo que necesitaban y el resultado fue una gigantesca falsificación de la historia del pensamiento económico alemán.

Por cierto, tal parece que al principio los nazis creyeron poder sacar un segundo tipo de provecho de los economistas históricos, a saber: que les ayudaran en el diseño de las nuevas políticas económicas. Es una de las muchas ironías de la historia que los historicistas no pudieran darles esa ayuda, pero no por falta de entusiasmo y entrega a la causa, sino por falta de solidez teórica, por lo que los nazis tuvieron que recurrir a esa nueva camada de economistas que se gesta en los años 30 en Europa y Estados Unidos: los que confían en la posibilidad de planificar la economía mediante la aplicación de las matemáticas y la estadística. Ni los economistas clásicos y neoclásicos, ni los economistas nacionales sirven a los nazis para sus propósitos de subordinación de la economía a la política; en cambio, las nuevas ideas de control de la economía que asociamos emblemáticamente con el nombre de Keynes proporcionaban ya entonces una "tercera vía". Sobre el tema volveré más adelante.

Pero volviendo a la colaboración entre nazis e historicistas, uno de los detalles más curiosos y reveladores que muestra la falsificación de la historia del pensamiento económico alemán es el hecho de que la escuela histórica alemana tenía una profunda vocación antinaturalista: para ella eran anatema tanto el utilitarismo y su obsesión con el "cálculo felicífico" de placeres y dolores, como el socialdarwinismo y su obsesión con la "supervivencia de los más aptos" y otras ideas evolucionistas, o el pragmatismo y su obsesión con las ciencias naturales y sus aplicaciones tecnológicas. Sin embargo, la idea central del nacionalsocialismo era justamente la de "raza" y la de "pueblo" como entidad biológica, es decir, era una idea ajena completamente al historicismo y en muchos sentidos opuesta a él. Janssen busca y busca en los más connotados autores de la escuela histórica alemana o de los aún más antiguos pensadores nacionalistas (como Fichte, Adam Müller o Friedrich List) y no encuentra rastro de semejantes ideas biologicistas. Éstas comienzan a aparecer apenas como consecuencia del intento de ciertos académicos de acercarse al régimen de Hitler.

No debe creer el lector que los historicistas estaban exentos de racismo (y particularmente, de antisemitismo). Antes bien, compartían ese tipo de prejuicios con la mayoría de la población alemana, y más generalmente europea, de la época. Pero Janssen muestra que, con la posible excepción de Sombart, estos prejuicios no eran parte integral y sistemática de sus investigaciones y de la ciencia que estaban tratando de construir. Por cierto, Max Weber sale bastante airoso del examen a que lo somete nuestro autor. Debo decir que en este punto no puedo estar totalmente de acuerdo con Janssen, ya que escritos como la lección inaugural 
que pronuncia Weber al asumir su cátedra en Friburgo no me parecen contener meros arrebatos emocionales, sino que, con cabeza bastante fría, Weber analiza el movimiento demográfico polaco frente al alemán y en ese contexto habla de "razas inferiores" . La controvertida interpretación del proyecto teórico de Weber que ha ido presentando Wilhelm Hennis a lo largo de los últimos 20 años apunta también en este sentido 5 . Pero la cuestión es demasiado espinosa para tratarla aquí más en detalle.

Resumiendo, pues, debemos evitar caer en la trampa de creer que una tradición tan larga y compleja como la de la teoría económica en Alemania puede entenderse simplemente como una preparación para el nacionalsocialismo, o bien que éste es la consecuencia lógica y el punto de culminación de aquella tradición. Para ello, debemos recordar que el racismo como un componente de la ideología nazi no era un elemento teórico de la Escuela Histórica Alemana; e, igualmente, que los nazis no tenían ningún interés en la ciencia histórica como tal. Al mismo tiempo, no debemos olvidar que la colaboración de muchos académicos - por cierto, no de todos- está en función directa de las intrigas políticas universitarias, y, en consecuencia, que tal colaboración dista mucho de ser una parte realmente poderosa del movimiento político en cuestión, ello a pesar de las ilusiones que más de algún académico pudo alimentar en su seno de pura falta de sentido práctico. Sobre esto se ofrecen más detalles en el segundo estudio.

\section{SEGUNDO ESTUDIO}

La Gleichschaltung de la ciencia económica, 1933 (Janssen, cap. 3, pp. 153222)

En la lengua alemana la expresión Gleichschaltung proviene de la ingeniería eléctrica y se refiere al acoplamiento (Schaltung) de flujos distintos de energía eléctrica de manera tal que se hagan iguales (gleich). Durante el régimen nacionalsocialista este concepto técnico se utilizó metafóricamente para referirse a la "igualación" de ideas y actitudes que requiere un régimen totalitario. El segundo estudio en el libro de Janssen pretende discernir qué pasó en el caso de la teoría económica; ya vimos en el primero que hay muchas diferencias entre la tradición

\footnotetext{
4 'El Estado nacional y la política económica', en: Gesammelte politische Schriften, 1921. Sin embargo, debe quedar claro que Weber -a diferencia de muchos otros académicos de su época-nunca fue antisemita, como lo confirma también la más reciente biografia (Michael Sukale, Max Weber: Leidenschaft und Disziplin, Tubinga, Mohr Siebeck, 2002).

${ }^{5}$ Cf. Max Webers Fragestellung: Studien zur Biographie des Werks, 1987; Max Webers Wissenschaft vom Menschen: Neue Studien zur Biographie des Werks, 1996; Max Weber und Thukydides: Nachträge zur Biographie des Werks, 2003 (todos publicados por Mohr Siebeck en Tubinga).
} 
historicista previa y las ideas de los nazis, pero la pregunta es ahora si los nazis lograron acoplar y ajustar las doctrinas tradicionales de manera que realmente esas diferencias desaparecieran.

La detallada y meticulosa investigación de Janssen pone en escena una verdadera comedia de errores. Recordemos que tenemos enfrente dos grupos de personas completamente divergentes. Por un lado, tenemos una clase política especialmente brutal y carente de escrúpulos y, por el otro, una clase intelectual entrenada para leer y escribir, investigar y dar clase, pensar y razonar. Los valores de uno y otro grupo no tienen realmente común denominador. Hasta lo que parece unir ambos grupos, a saber, la política, en realidad los separa: la política universitaria es a la política propiamente dicha lo que la música militar a la música propiamente dicha. De esa manera, los economistas de inspiración historicista pensaban que con ayuda de los nazis lograrían, primero, eliminar a sus enemigos - los economistas teóricos- de las posiciones universitarias importantes, con lo que, en segundo lugar, podrían recuperar la hegemonía de que habían gozado durante el siglo XIX y acaparar los apoyos financieros y, en tercero y último lugar (nótese la hybris), que podrían incluso convertirse en el verdadero poder detrás del trono. No puedo resistir la tentación de citar un pasaje en el que el autor ilustra la ingenuidad de los profesores con respecto al último punto:

\begin{abstract}
Muchos profesores creian además que tendrian éxito con el tiempo en pulir las groseras aristas de los nuevos señores. Asi por ejemplo, informaba Salin [un profesor de la época] sobre el ministro prusiano de finanzas Johannes Popitz [otro profesor de la época] haber alimentado durante dos años la esperanza de "poder educar al jefe de ministros [iningún otro que Hermann Göring!], convirtiéndolo en estadista a fin de usarlo luego para arrancarle los dientes venenosos al régimen". A tan "pueril opinión", declaró Hitler en 1938, a saber, que la burguesía "estaría en posición de apoderarse un dia intelectualmente" del partido, propinaron los nazis muy pronto una buena tunda (p. 156).
\end{abstract}

Es difícil creer en la distancia que los académicos hayan podido combinar de esa manera inocencia y arrogancia ${ }^{6}$. Janssen nos informa en una nota que Popitz (un hombre que combinaba la política y la academiá) sería eventualmente ejecutado por su posterior participación en la resistencia.

\footnotetext{
${ }^{6} \mathrm{La}$ combinación no es exclusiva de los economistas. Un caso muy significativo es el de Martin Heidegger, profesor de filosofia y rector de la Universidad de Friburgo (Janssen se refiere una vez a él como rector de la de Heidelberg, pero se trata obviamente de un mero descuido). Heidegger, como algunos economistas que cita Janssen, creyó también ser capaz de dominar y orientar el movimiento nacionalsocialista. Cuando años después le preguntan cómo pudo haberse engañado de tal manera, su respuesta es candorosa: aus Dummheit, 'por estúpido' (cf. Frédéric de Towarnicki, $\dot{A}$ la
} 
Los nazis, por su parte, también tenían tres cosas en mente: pensaban, primero, que los profesores les servirían para deshacerse de una serie de académicos incómodos, en segundo lugar, que podrían con su auxilio legitimar el régimen aludiendo al pasado glorioso de la investigación historicista y defendiendo sin trabas ni ambages cualquier política que ellos decidieran, y, finalmente, que los profesores producirían las investigaciones necesarias para lograr enderezar la economía de manera que se pudiesen cumplir sus objetivos de guerra, conquista, movilización total y dictadura. El chasco fue mayúsculo para ambas partes.

Un examen de los tres objetivos que se proponian los profesores y los tres que se proponían los políticos muestra que solamente había coincidencia real en uno: eliminar, o al menos neutralizar, todos aquellos elementos en la academia que estorbaban tanto a los nazis como a los historicistas, aunque por diferentes razones. ¿Quiénes eran estos? En orden de odiosidad: los judios, los marxistas, los socialistas no nacionalistas, los liberales y los teóricos. Es una lista curiosa, y, por supuesto, contiene múltiples traslapes: prácticamente todos los liberales eran teóricos, aunque algunos teóricos eran socialistas y hasta marxistas; prácticamente todos los marxistas eran internacionalistas, aunque algunos internacionalistas tenían más bien una inspiración cristiana y teológica; había judios de varias persuasiones, etc. En todo caso, a los economistas de la escuela histórica les estorbaban casi todos en cuanto que ocupaban plazas y puestos administrativos en las facultades; muchos economistas jamás hubieran podido hacer carrera si no hubiese habido tal número de expulsiones, arrestos y ejecuciones. Lo sorprendente aquí es que tantos y tantos académicos hubiesen aceptado métodos tan espantosos y tan alejados de la vida universitaria. Como dijo Hayek: "la devoción con la que los profesores e investigadores alemanes se pusieron a disposición de los nuevos gobernantes es uno de los espectáculos más abrumadores y vergonzosos de toda la historia del ascenso del nacionalsocialismo" (The Road to Serfdom, Londres, 1944; citado por Janssen, p. 153). Este solo hecho pone en duda la estatura moral de los intelectuales alemanes; pero no debemos olvidar que el apoyo irrestricto que muchos intelectuales europeos, estadounidenses y latinoamericanos proporcionarían después a los regímenes comunistas arroja el mismo género de duda.

rencontre de Heidegger, París, Gallimard, 1993). Sobre el tema general de semejante ceguera de los académicos ante las realidades de la política, recomiendo al lector la reciente obra de Richard A. Posner, Public Intellectuals: A Study of Decline, Harvard University Press, 2002. El recientemente depuesto Secretario de Relaciones Exteriores de México, Jorge Castañeda, tan reconocido como escritor y profesor cuanto fracasado como diplomático, es un ejemplo reciente de lo mismo; pero dudo mucho que él o ningún otro intelectual escarmentarán por ello. Después de todo -y esto son verdaderamente ya palabras mayores-, ni el propio Platón escarmentó, sino que luego de haber sido encarcelado y vendido como esclavo por el propio tirano al que quiso asesorar, continuó hasta su muerte planeando modos en que los filósofos pudieran gobernar. 
RIS

REVISTA INTERNACIONAL DE SOCIOLOCIA

№ 35, Mayo-Agosto, 2003

FERNANDO LEAL CARRETERO

Dada la coincidencia en el primer objetivo de cada grupo, éste se llevó a cabo más o menos a satisfacción de todos. En cambio, ninguno de los otros objetivos se cumplió realmente. El objetivo de legitimación mediante el historicismo requirió de la falsificación de los hechos históricos. De hecho, el principal resultado del primer estudio de Janssen es, como vimos, que la propaganda nazi nos sigue influenciando hoy hasta el punto de habernos "tragado" la historia de que la doctrina económica alemana de corte historicista, institucional y romántica tiene su culminación lógica en las ideas nacionalsocialistas. Algunos de los profesores se resistieron a esta "igualación", pero débilmente y sin éxito. Luego no podemos decir que esto satisfizo realmente su propósito propiamente científico de continuar la obra de esta tradición. El apoyo financiero y de otro tipo que el gobierno de Hitler les brindó fue insuficiente, errático y arbitrario. Por su parte, los profesores apoyaron en general, de palabra, las políticas del régimen, pero no pudieron realmente contribuir con investigaciones a las mismas. Una de las grandes ironías de toda esta historia es que finalmente fue un tipo de investigación distinta a la de los historicistas la que fue más utilizada por los nazis: la investigación macroeconómica y econométrica. Y con ello comenzamos a tocar la cuestión más importante: ¿qué clase de doctrina económica surgió realmente en la Alemania totalitaria después de la consolidación del régimen? A ella se aboca el tercero, más extenso y más importante de los estudios de este volumen.

\section{TERCER ESTUDIO}

\section{Surgimiento y desarrollo de una nueva doctrina económica en Alemania} después de 1933 (Janssen, cap. 4, pp. 223-535)

Es perfectamente comprensible que el lector de este libro de tanto en tanto se impaciente con tantos y tantos nombres y detalles, y se pregunte sobre la substancia propiamente doctrinal, sobre las concepciones económicas mismas que se debatían. Está muy bien enterarse de las intrigas y los pactos, de los engaños $y$ las ilusiones, de las traiciones y las zancadillas en que incurrieron los políticos criminales del 'tercer Reich' o sus académicos politicantes; pero también tenemos una justificada curiosidad por las ideas que estaban en juego y, eventualmente, por su uso y su eficacia en la práctica. Sin ceder en su afán histórico de proporcionar detalles y aclarar sutiles diferencias entre los actores, Janssen dedica más de trescientas páginas a este tema. Los tres estudios del volumen que nos ocupa aquí tienen ciertamente un orden cronológico: el primer estudio trata de los antecedentes doctrinales previos a la usurpación nazi, el segundo estudio, del intento de "poner a ritmo" la academia con la política durante el año de la toma de poder, y el tercero, de la consolidación de un cuerpo de doctrina distintivo del nacionalsocialismo después de ese año fatídico ${ }^{7}$. Sin embargo, falsificaríamos 
este libro si no viésemos que, aparte del orden cronológico, hay un orden sistemático: recuerde el lector que Janssen se propone responder a la pregunta de si la doctrina económica nazi es una culminación lógica del historicismo institucionalista alemán. Su respuesta es que no; que hay sin duda afinidades, pero que una conclusión afirmativa corre el riesgo de confundir cosas distintas y sustituir la comprensión científica por una satisfacción ideológica grosera: por decirlo así, hacer leña del árbol caído. Esta ha sido la actitud más frecuente en las investigaciones anteriores al libro de Janssen, y con ellas quiere el autor romper una lanza. Ahora bien, desde el punto de vista de las ideas, los dos primeros estudios apenas nos esbozan la situación, pero nunca acabamos de ver en concreto y de manera suficiente cuáles son los puntos doctrinales donde el nazismo se separa del historicismo tradicional.

El tercer estudio del libro de Janssen contiene tres partes: la primera, dedicada a la politica social (un nombre que expresa las intenciones profundas de la escuela histórica alemana), la segunda, a las concepciones sobre el dinero y los ciclos económicos (el tema fundamental de todos los economistas durante la crisis financiera de los años 30 , y por tanto, componente infaltable de los debates en la Alemania nazi), y la tercera y última, que presenta los elementos de la "nueva doctrina económica" buscada y alimentada por el régimen de Hitler. Mi exposición de todo ello será necesariamente comprimida y esquemática.

El mensaje esencial de la primera parte sobre la política social (pp. 225-289) muestra que, cuando se entra verdaderamente en detalle y se hace un análisis diferenciado de la situación doctrinal, aparece con claridad que los nazis se valieron de un cierto grupo de académicos para obtener alguna legitimación y eliminar a profesores ideológicamente indeseables, pero que, a fin de cuentas, lo que los nazis querían era bastante distinto de lo que buscaban los historicistas. La concepción doctrinal básica de la Escuela Histórica Alemana - el pilar de lo que podríamos llamar su teoría de la organización industrial- era la "comunidad empresarial" (Betriebsgemeinschaft), en la que los distintos miembros de la empresa se concebían de manera solidaria - de forma parecida a como hoy se habla, reinventando la rueda de la empresa como una gran familia, en la que, conscientes todos de estar "en el mismo barco", dejan de lado una actitud conflictiva para solucionar los problemas, atajar las diferencias, planear las estrategias y decidir la distribución de los ingresos de manera colegiada y solidaria. Es un cuadro muy bonito, surgido de las experiencias de unión, fraternidad y mutua dependencia de los soldados y

\footnotetext{
${ }^{7}$ Hablar de orden cronológico debe tomarse aquí cum grano salis, ya que Janssen es un cultivador del flashback narrativo a la manera de Joseph Conrad: puede comenzar una sección en cualquier lugar del tiempo, para luego adelantar y retroceder según le parece conveniente. Es una técnica interesante, aunque a veces algo fatigosa.
} 
Cientifica: la economía es una ciencia empírica y rigurosa, y la política económica y social debe basarse sobre los resultados de la investigación científica.

Relativista: los valores dependen de la situación histórica y son el resultado de la investigación científica inductivista; todo valor absoluto es producto de una especulación ilusoria.

Objetiva: aunque los valores cambian según las situaciones, eso no quiere decir que sean meramente subjetivos; los resultados axiológicos de la ciencia son rigurosos.

Nacionalista: la política social pretende ayudar a construir una nación aleman fuerte, llamada a ocupar un lugar muy importante en los asuntos mundiales.

Histórica: la economía no es una ciencia teórica, sino histórica; sus métodos y resultados son los propios de la historia; es perfectamente distinta de las ciencias naturales.

Pacifica: la política social emana de las experiencias de solidaridad de la guerra y pretende emular esas experiencias durante la paz ("economía de guerra durante la paz").

Igualitaria: la política social busca eliminar los conflictos de clase mediante métodos que permitan soluciones consensuadas entre el capital y el trabajo sobre la base del mutuo respeto.

Idealista: la economía y la política social podrán ser antisemitas, pero sólo en el sentido de buscar la superación de ciertas instituciones (p.ej. la usura, el agio y la especulacion), no la eliminación de individuos por su raza o religion (o sea, los judíos).

Corporativista: hay que tratar de implementar un tipo de economía en que cada rama de la producción constituya un estamento especializado y autónomo, de tal manera que el Estado sea un mero favorecedor de la actividad estamental y el parlamento sea subtituido por una cámara de los estamentos.
Dogmática: la economía y la sociedad deben construirse sobre la base de la voluntad férrea de los líderes y las concepciones que de ella surgen no son revisables por ciencia alguna.

Absolutista: los únicos valores que cuentan son los del nacionalsocialismo, que no proceden de una investigación, sino de la superior visión de su líder.

Pasional: los valores y la ideología nazis no son objeto de ciencia alguna; no se busca ni se pretende el rigor; nacen de la sangre (la pureza racial) y del sentimiento patrio.

Populista: la unidad que cuenta no es la nación, que es un mero accidente histórico, sino el pueblo (Volk) que es una unidad biológica racial, transhistórica y con demandas territoriales importantes.

Racista: la economía es una disciplina subordinada a una especie de darwinismo social que supone la existencia de razas superiores; su fundamento es biológico (hoy diriamos pseudo-biológico).

Belicosa: la política social no busca solamente una experiencia de solidaridad similar a la que se da en tiempos de guerra; busca la guerra misma.

Elitista: a pesar de una cierta demagogia obrerista, los nazis suponen que los jefes de empresa son superiores a los trabajadores; nada de igualdad en las negociaciones, sino principio de liderazgo.

Materialista: no se trata de ideales; los judíos constituyen no un tipo de cultura, sino una raza biológica que se opone en todo a la raza aria; como tal debe ser eliminada y liquidada mediante una política de 'limpieza étnica'.

Conduccionista: podrá haber un papel para los estamentos en la economía alemana, pero el Estado tiene poder supremo sobre todos los estamentos y la última palabra sobre todas las decisiones; la sabiduría de los líderes no se pone en cuestión por el saber técnico de la producción especializada. 
oficiales en el frente durante la primera guerra mundial. Las empresas convertidas en "comunidades empresariales" darían lugar - mediante una serie de mecanismos coporativistas, y sobre la base de un Estado estamentario ideal e idealizado- a una "comunidad del pueblo" (Volksgemeinschaft) que era, argumentaban los profesores, mucho mejor que la democracia caótica, verbosa e incompetente de la república de Weimar. Los nazis utilizaban toda esta terminología y retórica de los profesores historicistas con mucho gusto, si bien lo que tenían en mente era muy diferente e incluso opuesto. Podemos resumir estas diferencias y oposiciones en forma tabular, como se ilustra en el cuadro adjunto (ver p. 214).

Creo que el que estudie con cuidado este cuadro - cuyo apretado contenido está ampliamente explicado e ilustrado con nombres y apellidos en el libro de Janssen-no podrá ya tener duda de que, si bien puede decirse que el historicismo alemán "preparó el camino" para el nacionalsocialismo (y colaboró con su trabajo sucio dentro de las universidades), es imposible decir sin falsificación que sus concepciones doctrinales eran las mismas que las de los nazis, o que la política social nazi era el único camino posible a seguir por el historicismo alemán. De hecho, como Janssen observa en varios lugares, el sistema económico de la República Federal Alemana (la llamada 'economía social de mercado') es en muchos sentidos heredera de concepciones centrales de la Escuela Histórica. Finalmente, no debemos olvidar que no pocos profesores que se hicieron la ilusión de guiar y conducir la política social de Hitler fueron objeto de advertencias y reprimendas, prohibiciones y castigos, expulsiones y arrestos, justamente por empeñarse en sostener sus posiciones y, desde ellas, atreverse a criticar las políticas del régimen.

En cuanto a la segunda parte del tercer estudio (pp. 290-440), que se ocupa de la teoría monetaria y de la teoría de la coyuntura (ciclos económicos), el autor rompe una lanza contra la visión usual que pretende ver en la Teoría general de Keynes el punto de referencia obligado y único: los economistas alemanes del período en cuestión se ven entonces o bien como liberales irredentos, incapaces de entender la "nueva teoría económica", o bien como keynesianos avant la lettre (meros precursores de Keynes) y keynesianos sans lettres (diletantes que compartían sus opiniones económicas, pero incapaces del menor análisis teórico). Esta imagen, nos dice Janssen, está totalmente equivocada. Sin poner en entredicho la importancia y centralidad de Keynes, nos explica, en primer lugar, que la discusión germana en torno al papel del dinero, su relación con los ciclos económicos y el tipo de política que el gobierno debería implementar, es en parte, independiente de Keynes, por cuanto el atraso teórico inducido por la preponderancia del historicismo cedió después de la primera guerra mundial a los embates de los jóvenes economistas teóricos (Eucken, Rüstow, Lederer, Röpke, etc.), como se dijo en el primer estudio, con lo cual Alemania se puso rápidamente al día en materia de teoría económica. Durante los años 20, como es sabido, Alemania (al igual que Austria) entra en una grave crisis, para cuya explicación la Escuela Histórica no tiene nada que ofrecer, por lo que los teóricos, si bien de diferentes persuasiones, 


\section{RIS}

dominan muy pronto el debate profesional.

Durante ese debate el Keynes de The Economic Consequences of the Peace (1919) es ciertamente leído y celebrado, pero las líneas de la discusión monetaria no son propiamente anglosajonas: los nombres importantes son Menger, BöhmBawerk, Wicksell, Cassel, Schumpeter, Mises, Hayek. A mitad del debate se publica $A$ Tract on Monetary Reform (1923), el cual también tiene cierta influencia, aunque no es hasta el Treatise on Money (1930) cuando podemos empezar a hablar de una influencia realmente importante de Keynes sobre la discusión en el mundo de habla alemana. En cambio, la General Theory of Employment, Interest and Money (1936) aparece en un momento en el que, no solamente las posiciones en esa discusión están perfectamente dibujadas, sino en el que además, el principal problema que la Teoría general pretendía resolver, el desempleo masivo, ha sido vencido en Alemania. (En general, podemos decir que la gran crisis económica de principios del siglo XX aparece antes en Alemania que en los demás países industrializados, un hecho de gran importancia para entender la historia del pensamiento económico de la época). Se vio el libro como una notable síntesis de diversos aportes teóricos, pero no como algo fundamentalmente nuevo, revolucionario o de "grandeza clásica".

¿Qué forma había tomado el debate alemán de l'entre-deux-guerres? Para simplificar, podríamos ubicarlo en un espacio cartesiano de dos dimensiones: El eje horizontal correspondería a la teoría pura, el vertical, a la política. Si comenzamos con la política, podríamos situar en el extremo superior del eje vertical la posición liberal clásica (laissez faire laissez passer) y en el extremo inferior las varias posturas intervencionistas, que van desde el socialismo marxista hasta el diseño de políticas monetarias y coyunturales moderadas y transitorias, pasando por socialismos cristianos y solidarios, decisionismos estatistas y nostalgias del pasado prusiano. Los economistas liberales (tanto los viejos y nuevos austriacos, como los jóvenes economistas teóricos que surgen en la Alemania de posguerra) se encuentran todos, en principio, en el polo superior; pero a lo largo de la crisis económica una parte de ellos se va desplazando hacia el polo inferior, movidos por los avances de la teoría.

En cuanto a ésta, el polo derecho del eje horizontal sería la explicación de la crisis en términos de exceso de inversiones por parte de empresarios demasiado optimistas acerca de la abundancia real de capital, el cual a su vez es provocado por el exceso en la otorgación discrecional de crédito por parte de los bancos. Es fácil reconocer aquí las teorías económicas de la llamada Escuela Austriaca (Mises, Hayek). En cambio, el polo izquierdo de la teoría sería la explicación de la crisis en términos de fallas en el consumo (la "demanda agregada") como consecuencia del desempleo y a su vez causantes de desempleo debido a la desocupación del capital. El lector pensará enseguida en Keynes; pero independientemente de él, y a veces antes que él, encontramos en la Alemania de la época un sinnúmero de economistas que tienden a ver las cosas a la manera de Keynes. Algunos de 


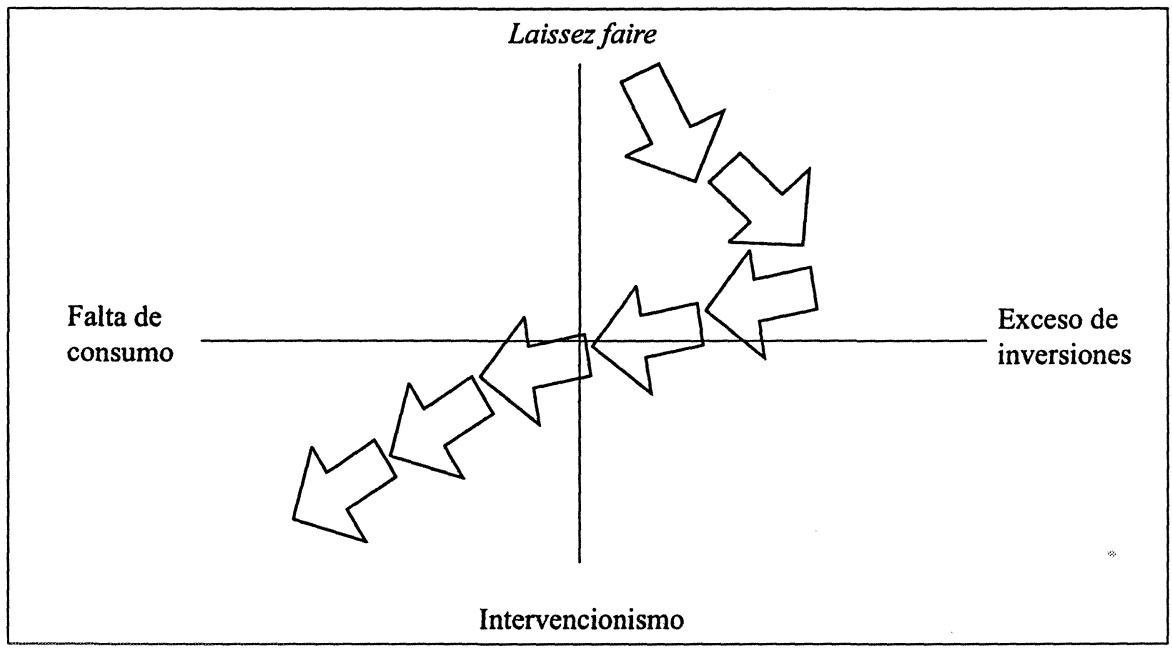

ellos son meros periodistas, diletantes y reformadores dominicales; otros son economistas más serios, dentro de la tradición del historicismo y el "socialismo de cátedra"; pero, según avanza la crisis, cada vez más teóricos de orientación, en principio liberal, comienzan a amistarse con ese tipo de explicación, o al menos consideran que, como lo dijo Eucken, "lo peor es que nada ocurra", es decir, que el gobierno deje que la economía siga su curso, si bien no están seguros de lo que habría que hacer. Para volver a la imagen bidimensional que propusimos antes, la dramática narrativa de Janssen nos muestra cómo la pérdida de fe en las explicaciones clásicas y neoclásicas de la crisis va produciendo un movimiento hacia el intervencionismo y una mayor confianza en una 'nueva doctrina económica' orientada hacia la demanda más que a la oferta, y hacia la discrecionalidad en el manejo del crédito más que hacia la disciplina del patrón oro (ver figura).

Pero ese movimiento a la vez teórico y político no se consolida: de hecho, la tesis principal de Janssen es que es justamente la indecisión por parte de los economistas acerca de unificar criterios y presentar propuestas concretas de intervención a la crecientemente débil democracia de Weimar uno de los factores que permitió a los nacionalsocialistas diseñar aquellas políticas económicas exitosas que les dieron tanta popularidad ${ }^{8}$. Tales políticas (y las teorías subyacentes) no

\footnotetext{
${ }^{8}$ Aunque Janssen es un autor admirable por la serenidad e imparcialidad con la que reporta los acontecimientos y las ideas, cabe decir que hay una ocasión en que sus preferencias teóricas y politicas hacen que describa la posición de Ludwig von Mises con respecto a la quantity theory of money de manera sesgada y poco diferenciada (pp. $315 \mathrm{~s}$.). Dejo aquí constancia de ello sin insistir ni elaborar más el asunto, tanto por falta de espacio, como por tratarse de un desliz menor.
} 
fueron realmente inventadas por los nazis, cuyos programas se movían entre la vaguedad y el delirio, pero fueron adoptadas de las que surgieron en el debate académico inconcluso de los años 20 y comienzos de los $30^{\circ}$.

Finalmente, la tercera parte (pp. 441-535) se ocupa justamente de la extraordinaria historia de la nueva doctrina económica alemana y sus peripecias a lo largo del régimen de Hitler. Janssen divide su fascinante narrativa en dos secciones: la primera, sobre teoría en sentido más estricto y la segunda, sobre política económica. Es, como nos advierte, una división algo artificial, pero muy necesaria para el propósito central de la obra: ver hasta dónde podría estar justificado decir que la nueva doctrina económica es la culminación lógica del historicismo alemán decimonónico. En cuanto a los aspectos teóricos, Janssen muestra que a cada uno de los principales elementos de la teoría keynesiana corresponde una teoría autóctona alemana:

\begin{tabular}{|c|c|}
\hline KEYNES & TEÓRICOS ALEMANES \\
\hline Teoría del empleo & $\begin{array}{l}\text { Teoría de la depresión secundaria y del } \\
\text { desequilibrio con diversos grados de empleo }\end{array}$ \\
\hline Teoría del multiplicador & $\begin{array}{l}\text { Teoría de la puesta inicial en marcha } \\
\text { (Initialzündung) y sus efectos primarios y } \\
\text { secundarios }\end{array}$ \\
\hline Teoría monetaria de la producción & $\begin{array}{l}\text { Teoría del crédito adicional y de la creación de } \\
\text { crédito productivo }\end{array}$ \\
\hline
\end{tabular}

Las propuestas teóricas de la columna derecha se remontan en algunos casos a la clásica Teoría del desenvolvimiento económico de Schumpeter (1911) y fueron alcanzadas por los economistas alemanes (Hahn, Röpke, Grünig, Lautenbach, Donner, Preiser, Föhl), o bien independientemente de Keynes, o bien bajo la influencia de obras anteriores a la Teoria general. Juntas constituyen realmente una nueva doctrina económica, radicalmente distinta de las teorías clásica y neoclásica, así como del liberalismo que va usualmente asociado a ellas. Sobre la base de esa doctrina es posible mostrar (a) que el capitalismo puede caer en una crisis de la que no puede salir por su propia dinámica, sino también que (b) ello requiere de una intervención del gobierno que combata el desempleo y saque a los empresarios del marasmo, y por cierto a través de (c) la creación de capital mediante la

\footnotetext{
${ }^{9}$ En este contexto resulta irónico que Keynes haya sido en general rechazado por los autores nacionalsocialistas, siendo él mismo quien había indicado que su teoría "se podía adaptar mucho más fácilmente a las condiciones de un Estado totalitario" que la teoría liberal (prefacio a la edición alemana de la Teoria general; citado por Janssen, p. 300, n. 314).
} 
emisión de dinero y la prestación de crédito más allá del ahorro real.

Si bien al principio, como dijimos antes, estas ideas encontraban cada vez más aceptación, según la crisis económica se agudizaba y el capitalismo no parecía salir por sí mismo de ella, no había acuerdo completo entre los economistas. Cuando los nazis llegan al poder, dejan todas esas disputas académicas de lado y sencillamente inician una intervención masiva en la economía y obtienen un éxito tan resonante y completo (reduciendo el desempleo de un 30\% en 1932 a un $8 \%$ en 1936) que la mayoría de los profesores aceptan la nueva doctrina. Pero para los nacionalsocialistas la lucha contra el desempleo y la revivificación de la producción no eran sino un medio para un fin: la economía bélica. Los teóricos irían viendo y viviendo esa transformación de la política social en política militar expansionista a lo largo de los años 30 . La actitud de la mayoría de ellos fue la de "cojear detrás de los hechos", buscando explicaciones doctrinales para las prácticas nacionalsocialistas. Esas prácticas se fueron haciendo cada veż más extremas, coercitivas y violentas, convirtiendo poco a poco la economía alemana en una economía centralmente planificada. Las voces críticas eran pocas, débiles $\mathrm{y}$, por razones obvias, cautelosas e indirectas. Janssen relata con exquisito detalle las piruetas y acrobacias teóricas que los profesores iban inventando para explicar, justificar o simplemente ensalzar los excesos cada vez mayores en que incurrían los nazis al ir eliminando todas las libertades económicas del pueblo alemán en aras de una presunta "voluntad comunitaria". ¿Que hay límites a la expansión crediticia por parte de las demás naciones? Solución: la magra autarquía sobre la base de los recursos naturales de Alemania dará paso a una nueva autarquía basada en la conquista de nuevos territorios y sus abundantes recursos. ¿Que hay límites al crecimiento de la deuda pública, la cual tarde o temprano se tendrá que pagar? No importa: el botín de las tierras conquistadas servirá para pagar todos los bonos emitidos. ¿Que hay límites debido a la inflación y el mercado negro? Nada de eso: el mundo se asombrará de los controles de precios, salarios y ganancias que los nazis son capaces de poner en obra. Ya Hitler le había dicho a Rauschning:

Hay inflación cuando se quiere que haya inflación. La inflación es falta de disciplina. Falta de disciplina de compradores y falta de disciplina de vendedores. Ya me haré cargo yo de estabilizar los precios. Para eso tengo mis tropas de asalto. (Citado por Janssen, p. 108).

Y unos años después añadiría: "La causa principal de la estabilidad de nuestra moneda son los campos de concentración" (citado por Janssen, p. 526). Con estos métodos todo es posible, al menos por un tiempo ${ }^{10}$. Esto no sorprende tratándose

\footnotetext{
${ }^{10}$ Por lo demás, no mucho, ya que, como el propio Hermann Göring, encargado final del control de precios, atestiguara tras la rendición, este método secular había sido tan inefectivo en la Alemania
} 
de un dictador. Lo que sorprende algo más son los intentos por parte de los académicos de creer que todo esto constituía un nuevo tipo de sistema económico, una tercera vía, distinta del socialismo y del capitalismo, y para la que "sólo los alemanes estaban preparados" "1.

La conclusión de Janssen es que el sistema económico de Hitler era, en efecto, distinto del socialismo y del capitalismo, pero no en el sentido de las especulaciones de algunos economistas que hablaban de "economía dirigida" por oposición a "economía libre" y "economía planificada". Más bien en el sentido de una economía de guerra, por la guerra y para la guerra, una economía dirigida a la destrucción y a la conquista, una "economía forzada" (Zwangswirtschaft).

El balance de todo lo anterior aparece en las brevísimas páginas de la conclusión (pp. 537-539): la teoría clásica y el liberalismo que habían florecido durante la república de Weimar son derrotados y dejados a un lado en las universidades; pero la Escuela Histórica, si bien es utilizada al principio, resulta de poca utilidad para los nazis y por lo tanto, aunque manteniendo algunas cátedras, pasa a un segundo plano frente a la 'nueva doctrina' que se forma de elementos de los dos adversarios, enormemente transformados ante la necesidad de explicar los fenómenos de la inflación galopante y el desempleo masivo de los años 20 y 30 . Los hechos, pues, no autorizan a pensar que el pensamiento económico alemán tiene características especiales y ha seguido un camino histórico sui generis. Ahora bien: la 'nueva doctrina' está en armonía con la fuerte intervención estatal en contra del desempleo y por la reanimación económica; pero no está realmente en armonía con el motivo subyacente a ese intervencionismo, a saber, la política expansionista y beligerante de los nazis. Por más esfuerzos que algunos académicos (cada vez menos según pasaba el tiempo y siempre los menos talentosos) hayan hecho por crear una teoría específica del sistema económico totalitario, la 'nueva doctrina' no era ella en sí misma ni totalitaria ni militarista.

El gran mérito del libro de Janssen es justamente su capacidad y paciencia para rastrear los detalles biográficos y bibliográficos de las distintas personas involucradas, de manera que establece quién era quién y quién pensó, dijo o escribió

\footnotetext{
nazi como en los siglos que lo precedieron (R. Schuettinger y E, Butler, Forty Centuries of Wages and Price Controls, Washington, Heritage Foundation, 1979, p. 73).

"Janssen, p. 533. La frase es de Erich Preiser, viejo socialista y uno de los representantes de la "nueva doctrina económica". Y si bien ella contiene ecos indudables del historicismo (la especificidad germana), debemos recordar que si Alemania respondió tan rápidamente a las opresivas políticas económicas de Hitler, ello se debió a que una buena parte del aparato intervencionista había sido creado previamente por el gobierno socialdemócrata de Weimar (Schuettinger y Butler, op. cit., p. 66).
} 
qué, con lo cual combate esos juicios fáciles tan comunes en un área de estudio tan propicia a los prejuicios, las pasiones y la superficialidad como son los vínculos entre la academia y el nazismo. Se trata de un correctivo excelente contra tantos y tantos investigadores en la República Federal Alemana que se han dado golpes de pecho público, han exhibido su political correctness, y han hecho carreras enteras sobre la base de desprestigiar a los académicos de la época pretendiendo demostrar que sus obras "contienen en germen" y "conducen inevitablemente" a los campos de concentración y a las cámaras de gases. Aparte de entender muy poco la verdadera relación entre la investigación y la política (ésta se vale de aquélla, pero aquélla tiene escasísima influencia sobre ésa), confunden el chisme y la maledicencia con el estudio histórico serio. Lo cual, por cierto, no quita que muchos de los académicos a las que les tocó vivir, trabajar y hacer carrera en aquella época tan desdichada se hayan portado en muchas ocasiones de manera desvergonzadamente oportunista y lamentablemente cobarde. Sobre esta ultima ratio, sin embargo, no queda otra que recordar aquello de que "el que esté libre de culpa, que lance la primera piedra". Cuando Karl Jaspers, consternado por la usurpación nacionalsocialista y por lo que ya prometía en su antisemitismo furibundo, le dijo a Martin Buber (pensador judío) que le preocupaba qué haría éste ahora, Buber con toda razón le replicó: "Lo interesante no es qué voy a hacer yo, sino qué va a hacer usted." A los lectores que se inclinan fácilmente a los juicios morales les preguntaría yo si creen de veras que en igualdad de circunstancias ellos se habrían comportado de manera más heroica y admirable que los diversos académicos estudiados por Janssen. Como dice Conrad en Lord Jim, nadie sabe de qué tamaño es su valor o su cobardía hasta que llega el momento en que la cuestión se pone a prueba.

Y ni siquiera la explicación por cobardía resulta cierta o completa. Cuentan que en una reunión de los altos mandos del FBI en que se hablaba una vez más de los interminables conflictos entre esa oficina y la Casa Blanca, el célebre J. Edgar Hoover atajó a los quejosos diciéndoles poco más o menos: "No hagan Uds. tanto caso de los Kennedy. No son gente importante. Los políticos van y vienen, según los azares de las elecciones. Nosotros, en cambio, permanecemos." Estas palabras, tan reveladoras sobre la verdadera relación entre políticos electos y burócratas establecidos, podrían arrojar también alguna luz sobre los motivos profundos de las acciones de los profesores universitarios durante el nazismo. Desde que Tucídides declaró haber creado un ktêma eis aei hasta la novísima aparición de la big science, los investigadores han tenido siempre la íntima convicción que su obra colectiva es philosophia perennis, ciertamente "más duradera que el bronce". Adoptando, así, su usual perspectiva "bajo el aspecto de lo eterno", les parece claro - correcta o incorrectamente- que las peripecias de lo político tienen relativamente poca substancia. No resulta, por tanto, poco plausible pensar que, ante un Hitler y sus secuaces, más de alguno habrá dicho para sus adentros, como Hoover: "Ya pasarán". Sobre esa base no es en realidad extraño que hayan 
RIS

REVISTA INTERNACIONAL DE SOCIOLOCIA

№ 35, Mayo-Agosto, 2003

FERNANDO LEAL CARRETERO

estado dispuestos a compromisos que nos parecen ahora vergonzosos y hasta en algunas ocasiones innecesarios.

La editorial Metropolis de Marburgo (www.metropolis-verlag.de) tiene el gran mérito de haber venido impulsando el conocimiento histórico documentado de la historia de la ciencia económica alemana. Este libro de Janssen es una más de las importantes obras que debemos agradecer a ese loable esfuerzo educativo y aufklärerisch ${ }^{12}$.

${ }^{12}$ Tal vez el único defecto que afea la publicación es que la corrección de pruebas dejó bastantes errores tipográficos, especialmente de puntuación. Por lo demás, el libro está muy bien impreso y encuadernado. 